A N N A L E S Annales de Bretagne et des Pays de l'Ouest

\title{
Les tentatives de regroupement des droites dans les années trente
}

Jean Vavasseur-Desperriers

\section{OpenEdition}

Édition électronique

URL : http://journals.openedition.org/abpo/1556

DOI : $10.4000 /$ abpo. 1556

ISBN : 978-2-7535-1487-4

ISSN : 2108-6443

Éditeur

Presses universitaires de Rennes

Édition imprimée

Date de publication : 20 septembre 2002

Pagination : 61-77

ISBN : 978-2-86847-768-2

ISSN : 0399-0826

\section{Référence électronique}

Jean Vavasseur-Desperriers, "Les tentatives de regroupement des droites dans les années trente », Annales de Bretagne et des Pays de l'Ouest [En ligne], 109-3 | 2002, mis en ligne le 20 septembre 2004, consulté le 19 avril 2019. URL : http://journals.openedition.org/abpo/1556 ; DOI : 10.4000/abpo.1556 


\title{
Les tentatives de regroupement des droites dans les années trente
}

\author{
Jean VAVASSEUR-DESPERRIERS \\ Professeur d'histoire contemporaine, Université de Lille 3 \\ Centre de Recherches sur l'Histoire de l'Europe du Nord-Ouest
}

On sait que la dispersion des forces de droite, qui a longtemps caractérisé de façon permanente la vie politique française, eut pour constant corollaire un effort inégal et jamais couronné de succès en vue de regrouper ces éléments séparés tant par les rivalités personnelles que par des divergences idéologiques ou stratégiques.

Il convient de délimiter précisément l'objet de la présente communication. Il ne sera pas ici question des rapprochements parlementaires, dont le modèle restait dans l'entre-deux-guerres la Délégation des gauches au temps du Bloc des gauches. Sans atteindre l'efficacité de ce précédent datant du début du siècle, la droite connut dans l'entre-deux-guerres plusieurs tentatives de mise sur pied d'un intergroupe rassemblant la droite et le centre droit, d'abord sous la législature dite du " Bloc national " (19191924), puis sous le Front populaire, à l'initiative de la Fédération républicaine de Louis Marin (1936-1938). On ne s'en occupera pas ici, pas plus que des cartels, qui, tels ceux de 1928 ou 1936, visaient exclusivement des accords électoraux. Laissant de côté ces divers types de rapprochement qui mériteraient une étude spécifique et approfondie, l'attention se portera exclusivement sur les tentatives de type " confédéral " : on entend parlà une action de rapprochement d'organisations politiques en vue de déléguer à une structure commune un certain pouvoir d'initiative.

Notre attention se portera sur les expériences des années 1930. Il est toutefois indispensable d'évoquer les précédents des années 1920, durant lesquelles les droites avaient été tentées par des initiatives de ce type.

\section{La tentative des années 1920 : l'Action nationale républicaine}

Cette organisation se situait dans le prolongement du " Bloc national républicain ", rassemblement né dans les dernières semaines de 1919, à la veille de l'échéance électorale de novembre 1919. Mais, comme on le sait, ce rassemblement présentait un caractère à géométrie variable, cette déno- 
mination générique dissimulant des accords différents conclus entre des partenaires variables, la principale différence résidant dans la présence ou non du Parti radical. Ces accords, d'autre part, n'avaient donné naissance à aucune structure permanente. Enfin, et surtout, l'objectif du « Bloc national " était bien de prolonger l'union nationale née dans la guerre, et non de procéder à une union des droites, bien que les formations qui avaient accepté d'y participer pussent être classées majoritairement à la droite de l'échiquier politique.

\section{Une véritable organisation}

L'Action nationale républicaine (ANR) fut mise sur pied au début de 1922, alors que la formule du " Bloc national " arrivait à épuisement, les épisodes parlementaires ayant montré l'inexistence effective de cette coalition. En avril 1922, naissait l'Action nationale républicaine, dotée des attributs qui permettent de définir une véritable organisation : un comité directeur, un bureau, un manifeste programme, un secrétariat général, un bulletin à parution périodique.

Le premier se composait de 34 membres, majoritairement des parlementaires (21, dont 11 membres de l'Entente républicaine démocratique, 4 de l'Action républicaine et sociale, un du groupe des Républicains de gauche). Parmi les 13 non parlementaires, on pouvait distinguer les représentants d'organisations politiques - mentionnés en tant que tels dans la liste des membres des instances dirigeantes -, comme l'Action libérale populaire, les Républicains démocrates (démocrates chrétiens), l'Union nationale républicaine (groupe rassemblant les républicains d'origine plébiscitaire, tel Marcel Habert), la Ligue civique et la IV République (ces deux derniers groupes, nés au lendemain de la guerre, s'étaient donnés pour tâche de transformer les institutions parlementaires, mais leur " révisionnisme » n'avait pas rencontré l'écho espéré). D'autres personnalités étaient mentionnées comme appartenant au monde de la presse : un administrateur de L'Éclair, un dirigeant du groupe catholique La Presse régionale (Jules Dassonville), le directeur du Télégramme de l'Est (Ernest Pezet). Le président de la Fédération républicaine, Auguste Isaac, figurait parmi les viceprésidents de l'ANR ${ }^{1}$.

Le bureau, composé de 10 membres, obéissait lui aussi à ce principe " confédéral ». Il comprenait 5 parlementaires, dont le président François Arago, un des principaux leaders du centre droit, et 5 non parlementaires, dont le comte Xavier de La Rochefoucauld, de l'Action libérale, Alfred Bour, de la Fédération des républicains démocrates, et deux personnalités considérées comme des techniciens de l'organisation, chargés des tâches matérielles : Albert Orry, le secrétaire général, et Hubert Bourgin, le « chef de la documentation ". Avec ces deux derniers personnages, on se trouvait en présence d'un type politique particulier. Militant venu du socialisme,

1. L'Intransigeant, du 8 avril 1922. 
Bourgin avait activement œuvré en faveur d'une révision du système institutionnel de la III ${ }^{\mathrm{e}}$ République dans un de ces petits groupes actifs en 1919, la Ligue civique. Albert Orry avait milité au Parti socialiste national d'Alexandre Zévaès, indéfectiblement favorable au maintien de l'Union sacrée, pendant et après la guerre; il avait par la suite occupé le secrétariat général du Bloc national. La gérance du bulletin hebdomadaire du mouvement, intitulé sobrement L'Action nationale républicaine, dont le premier numéro parut le 12 octobre 1922, fut confiée à Albert Orry. On y trouvait deux catégories de rubriques, les unes concernant l'action de "nos amis " parlementaires, les autres relatant les manifestations des organisations membres de l'ANR.

On n'abordera pas ici les raisons politiques qui furent à l'origine de l'échec de l'ANR : le Parti républicain démocratique, formation du centre très attachée à la laïcité, et soucieux de conserver le contact avec les radicaux, refusa d'y adhérer. Les objectifs poursuivis par les diverses composantes de la " confédération " étaient par ailleurs beaucoup trop divers, sinon divergents : former un pôle républicain modéré libéral pour la Fédération républicaine, maintenir la spécificité de l'action des catholiques à l'heure de leur intégration à la République pour l'Action libérale, œuvrer en faveur d'une campagne "révisionniste " pour les techniciens de l'organisation. Tout cela ne pouvait que favoriser le déclin rapide de cette tentative à peine esquissée. On s'attardera surtout sur les aspects structuraux du problème.

\section{Un fonctionnement confédéral difficile}

Sur le plan organisationnel, une ambiguïté fondamentale subsistait sur la nature des rapports existant entre la confédération et les organisations adhérentes. Sur un point, la réponse ne peut faire de doute : les organisations adhéraient à l'ANR en tant que telles. Une note du secrétariat de la Fédération républicaine précise les associations adhérentes : outre celles citées plus haut, on relevait l'Association nationale pour l'organisation de la démocratie et la Ligue démocratique d'action morale et sociale ${ }^{2}$. Il ne semble pas que l'adhésion individuelle en dehors des associations ait été possible. Quelle pouvait donc être la réalité du pouvoir dans un tel type d'organisation? L'autonomie d'une telle association pouvait-elle se réaliser dans des prises de décision spécifiques, indépendantes des intérêts des organisations adhérentes? Auguste Isaac, président de la Fédération républicaine, répondit à cette question au cours d'une réunion tenue par son parti à la Brasserie Lutetia, en novembre 1922, quelques mois après la création de la confédération. Certains d'entre nous, dit-il, sont entrés, à la demande l'ANR, dans le comité directeur de cette organisation, mais cela " ne veut pas dire que nous ayons le moins du monde aliéné notre indépendance ${ }^{3}$ ".

2. Note du secrétariat de la Fédération républicaine, s.d. [1921], Papiers Marin, Archives nationales (AN), 317 AP 75.

3. La Fédération républicaine de novembre 1922. 
Un an plus tard, en novembre 1923, Auguste Isaac et le secrétaire général de la Fédération Jean Larmeroux étaient amenés à préciser leurs vues, à l'occasion d'une des dernières réunions tenues par l'ANR, privée depuis le mois de juillet précédent de président par suite de la démission de François Arago. Le président et le secrétaire général de la Fédération se firent mandater par le conseil général de leur parti pour déclarer à la réunion de l'ANR que " la Fédération républicaine ne saurait admettre en aucune manière d'être inféodée à un groupement constitué au-dessus d'elle et qu'elle tient à affirmer sa complète indépendance. " La Fédération déclarait ne voir dans l'ANR qu'un "intergroupe chargé de mettre en liaison les partis voisins et amis " - c'est nous qui soulignons. Dès lors, l'organisation se replia sur les tâches matérielles, les conférences ou la diffusion d'informations ${ }^{4}$.

Ainsi, l'échec de cette première tentative de confédération soulevaitelle des problèmes que l'on allait retrouver par la suite. La représentation des composantes, tout d'abord, et la question annexe de l'équilibre entre parlementaires et non parlementaires; celle des moyens matériels propres, sans lesquels il n'est point d'autonomie possible; enfin le pouvoir de décision réel de cette structure, par rapport à ses composantes.

\section{Le cas de la Ligue républicaine nationale}

La création de la Ligue républicaine nationale (LRN) en octobre 1924 par Alexandre Millerand, en réponse à la victoire des Gauches aux élections générales de mai 1924, sembla une réponse aux questions posées par l'échec de la " confédération " qu'avait été l'ANR. La Ligue créée par l'ancien président de la République se présentait comme une formation neuve, et non une confédération. Trois caractéristiques la différenciaient nettement de l'ANR. Il s'agissait tout d'abord d'une structure plus ouverte, puisqu'elle regroupait des adhérents " directs", non inscrits aux diverses formations membres de la Ligue, et des groupes affiliés, dont l'Alliance démocratique et la Fédération républicaine. L'appareil, d'autre part, était bien plus étoffé : la LRN disposait d'un secrétariat général, d'un directeur administratif, de délégués régionaux permanents. Enfin, dans l'esprit du fondateur de la Ligue, il s'agissait de rompre avec une structure confédérale. Le comité directeur formé par Millerand comptait 15 membres : 11 choisis parmi les dirigeants politiques (Antony Ratier, président de l'Alliance démocratique; Auguste Isaac, président de la Fédération républicaine; Louis Marin, président de l'Union républicaine démocratique, le nouveau nom de l'Entente dans la Chambre élue en 1924) et 4 parmi diverses personnalités du monde économique ou culturel ${ }^{5}$. Mais le président de la Ligue refusait de les considérer comme des représentants d'une association extérieure. À Isaac qui, en novembre 1924, ne pouvant assister à

4. Compte rendu du conseil général de la Fédération républicaine du 14 novembre 1923, AN, 317 AP 82.

5. Note Guiter, [oct. 1924], AN 317 AP 75. 
une réunion publique de la Ligue, proposait la nomination d'un de ses viceprésidents pour le remplacer au comité directeur de la Ligue, Millerand répondit que son organisation n'était pas une "émanation parlementaire des différents partis ${ }^{6}$ ". Il semble pourtant que les chefs des partis adhérents la considéraient comme telle; et ce fut là une des causes majeures de son échec et de sa mise en sommeil un an plus tard.

Ainsi, les années 1920 n'offraient guère de précédent convaincant aux tentatives d'unification des droites. Bien au contraire, chaque tentative avait pour effet de souligner les divergences entre les forces concernées et de mettre en valeur la spécificité des organisations.

\section{Un instrument de rapprochement des ligues dans les années 1930 : le Front national}

Un premier type de rapprochement est observable dans les années 1930, qui concerne non pas les partis, mais les ligues. En mai 1934, est lancé un appel publié dans L'Ami du peuple, en faveur d'une entente ouverte à toutes les associations "nationales " en vue de lutter contre les forces de la " révolution" regroupées dans le " Front commun ". Les signataires de l'appel provenaient de deux ligues, la Solidarité française du commandant Renaud et les Jeunesses patriotes de Pierre Taittinger, soit deux organisations ligueuses, caractérisées par le déploiement dans la rue des forces " nationales " face aux " révolutionnaires ".

\section{Des objectifs pratiques?}

Le Front national des années 1930 se constitua, nous indiquent les sources policières, sur des objectifs pratiques, dans un contexte de brutalisation de la vie politique. Les " organisations nationales ", remarquait un observateur de la Préfecture de police de Paris le 19 mai 1934, tendent maintenant à " se prêter mutuellement main forte pour se défendre contre les agressions qui semblent faire partie d'un plan arrêté de leurs adversaires ${ }^{7}$ ". L'argument semble à première vue discutable, surtout s'agissant d'un " plan arrêté " des troupes adverses. Chaque ligue du reste, disposait de son propre service d'ordre. Le problème de l'entraide se posait plutôt au niveau des partis parlementaires, qui, dépourvus d'organes de ce type, devaient faire appel à l'appui des mouvements ligueurs. Ainsi, la Fédération républicaine, et parfois des candidats "nationaux " indépendants, faisait appel aux Jeunesses patriotes pour la protection de leurs meetings. Il ne semble pas douteux que les organisations affiliées au Front national aient songé à étendre leur influence en protégeant l'ensemble des candidats " nationaux ${ }^{8}$ ".

6. Isaac à Millerand, 17 décembre 1924; Millerand à Isaac, 19 décembre 1924, Papiers Millerand, 470 AP 104.

7. Rapport du 19 mai 1934, Archives de la Préfecture de police de Paris (APP), BA 1964.

8. APP, 26 mai 1934, BA 1964. 
D'autres sources indiquent une raison différente, le souci d'étalage de la force, qui aurait joué un rôle prépondérant. Il s'agissait essentiellement de coordonner les démonstrations de rue, comme l'explique un observateur de la Préfecture :

"Si les groupements cherchent à briller individuellement, ils n'en sont pas moins désireux pour cela d'observer entre eux une certaine cohésion, de manière à impressionner le public par l'importance de leurs effectifs, et, par la même occasion, à intimider leurs adversaires ${ }^{9}$."

Cette explication par un souci de visibilité extérieure est plus convaincante, à condition de la situer dans le contexte de l'évolution du rapport de force entre les ligues. Les deux ligues mentionnées dans l'appel fondateur sont confrontées, dans ce tournant de 1933-1934, à une vive concurrence, celle des Croix-de-Feu. Les anciens combattants de l'Union nationale des combattants (UNC) mobilisent par ailleurs massivement en dehors d'elles. Leur regroupement permettrait donc des démonstrations d'importance, dont l'effet pouvait rejaillir sur chacune d'elles.

D'autres motifs, plus politiques, doivent être recherchés. Un important article de L'Action française, signé de Maurice Pujo, publié en mai 1937º, fournit une autre clé de la formation du Front national : il s'agissait avant tout, au départ, de nouer des liens placés "sur le terrain immédiat et pratique ", en évitant de poser les questions de fond, l'essentiel étant de parvenir à unir le plus grand nombre de militants possible, tandis qu'une " élite ", celle des dirigeants de l'Action française, ligue associée mais non adhérente, se chargerait d'élaborer et de propager la pure doctrine. On voit ici que l'alliance formée "à la base " ne pouvait se réaliser sur la base d'un contenu politique précis : les uns, Jeunesses patriotes et Solidarité française, tentaient de parer au déclin de leur influence, les autres, l'Action française, poursuivaient leur lutte pour étendre leur influence idéologique. On s'explique que la propagande du Front national soit restée purement négative, visant essentiellement le "Front commun " et la franc-maçonnerie ${ }^{11}$.

\section{Une structure légère}

Le Front national était doté d'un secrétariat général, confié à une personnalité venue du bonapartisme, Charles Trochu ${ }^{12}$. Industriel, bordelais d'origine (la Compagnie générale de la grande pêche), Trochu avait milité aux Jeunesses patriotes en même temps qu'à l'Appel au peuple. Mais il avait établi des liens personnels avec d'autres mouvances de la droite, par son appartenance aux Croix-de-Feu, et même à l'Alliance démocratique. Sans qu'une adhésion formelle à l'Action française ait été prouvée, il semble

9. APP, 12 mai 1934, BA 1964.

10. L'Action française, du 24 mai 1937.

11. APP, 24 décembre 1934, BA 1964.

12. Notice sur Trochu dans la thèse de Jean PhiLIPPET, Le Temps des ligues. Pierre Taittinger et les Jeunesses patriotes, IEP de Paris, 1999, Annexes, p. 96. 
certain qu'il entretenait des liens avec le mouvement royaliste. Un tel œcuménisme, sans qu'on puisse déceler une adhésion ferme et une conviction précise (les rapports attribuent à Trochu les inclinations les plus diverses : Appel au peuple, Action française, etc.), le désignait aux yeux des divers partenaires comme l'animateur de l'organe de liaison. Son élection au conseil municipal de Paris lors des municipales de mars 1935 renforça sa position au sein du Front national. Désigné dès le printemps 1934 comme secrétaire général, Trochu était secondé par deux secrétaires généraux adjoints, René Richard, un des dirigeants des Jeunesses patriotes proche de Taittinger, et Fromentin, par ailleurs secrétaire général de la Solidarité française ${ }^{13}$. Responsable de la propagande des JP, Richard prit en mains celle du Front national comme délégué général. La composition du bureau dirigeant montrait bien le caractère de l'" organe de liaison " : il s'agissait de coordonner des actions, non de s'engager dans des initiatives de fond. La position assez autonome de Trochu, toutefois, ne fut pas sans lui laisser une certaine marge, à vrai dire bien étroite.

Les moyens matériels attestent bien la nature de l'organisation et son absence d'autonomie. Pour faire face à des frais considérables - transport des membres des services d'ordre, affichage, location de locaux pour les réunions, toutes ces opérations étant assurées par l'organe de liaison -, le Front national n'avait pas à l'origine de budget propre. Les premières dépenses furent couvertes à titre personnel par Trochu ${ }^{14}$. Une souscription fut lancée par le commandant Renaud, chef de la Solidarité française en mai 1934 à l'adresse de tous les sympathisants de la ligue ${ }^{15}$. Mais il ne semble pas en définitive que le Front national ait pu réellement bénéficier de l'autonomie financière. Surtout, le service d'ordre était formé de manière composite, les militants des diverses organisations, Solidarité, Jeunesses patriotes et Action française, juxtaposant leurs troupes sans les fusionner. La coopération s'établissait toutefois de façon étroite pendant les réunions publiques ${ }^{16}$, Camelots et $\mathrm{JP}$ " combattant " alors au coude à coude. Ainsi se réalisait l'" union à la base " dans l'action " limitée et immédiate ".

\section{Une action de liaison}

Le Front national organisa sous son sigle de nombreuses réunions, particulièrement en 1934 et au début de 1936, avant les élections générales. Au printemps de 1936, la présence de l'organisation était remarquée en province et à Paris : cette époque marqua sans doute l'apogée de l'organe de liaison ${ }^{17}$. L'importance de la province dans son action mérite d'être soulignée et l'étude des rassemblements dans les villes de province, certaine-

13. APP, 13 juin, BA 1942.

14. APP, 23 juin 1934, BA 1964.

15. APP, 26 mai 1934, BA 1964.

16. APP, 2 juin 1964, BA 1964.

17. APP, 25 mars 1936, BA 1964. 
ment très nombreux, reste à faire. Peut-être que les différences entre « nationaux " étaient ici moins marquées que dans la capitale ${ }^{18}$.

Il faut toutefois souligner que le Front national, s'il pouvait prendre des initiatives propres, dépendait étroitement des réactions des organisations adhérentes. Ainsi adressa-t-il, dans le contexte de la campagne antimaçonnique de décembre 1934, une lettre à tous les parlementaires - à l'exception de ceux d'extrême gauche - pour leur demander des éclaircissements sur leur attitude face à la franc-maçonnerie. La lutte contre les projets législatifs envisagés contre les ligues et l'agitation de rue était également l'objet de mobilisation ${ }^{19}$. Toutes les organisations adhérentes au Front national signèrent la lettre, l'Action française qui n'en faisait pas partie la contresignant également ${ }^{20}$. Ainsi, le Front national ne s'exprimait-il en tant que tel que dans la mesure où ses composantes le lui permettaient.

Il est donc naturel que les questions politiques de fond - de " doctrine ", si l'on veut - aient été rarement traitées par la propagande du Front national. Au cours d'une réunion tenue par le Mouvement national populaire, mis sur pied par Taittinger à la fin de 1935, Charles Trochu devait souligner, en mettant avec insistance l'accent sur le rôle positif joué par les Jeunesses patriotes, qu'aucune question, " d'ordre général ou de politique générale " n'avait été traitée au Front national sans l'accord de Taittinger ${ }^{21}$. Aussi, la propagande se révélait-elle purement négative. Outre la dénonciation de la franc-maçonnerie, le Front national participa à la campagne antisanctionniste de la fin de 1935, comme l'atteste une réunion organisée salle Wagram en octobre de cette année. Mais la diversité des organisations associées dans le Front national lui interdit de devenir une force de proposition.

\section{Un lieu de rivalités}

Le Front national, on l'a vu, résultait de l'appel lancé par deux ligues, la Solidarité française et les Jeunesses patriotes. L'Action française s'associa à la création du Front, mais n'y adhéra pas formellement. Cependant, par la suite, ses troupes participèrent activement aux services de protection des manifestations et la ligue royaliste prit part aux diverses manifestations et défilés, notamment en faveur de Jeanne d'Arc, comme en mai 1934 et en mai 1935. Le refus d'adhérer au Front national fut sans doute volontaire. Il n'est pas exclu que la spécificité souvent répulsive, même dans le monde ligueur, de la ligue royaliste ait poussé les dirigeants du Front national à la maintenir formellement en dehors, avec d'ailleurs l'accord de la ligue elle-même, soucieuse de promouvoir avant tout l'unité dans l'action, sur le terrain "immédiat et pratique ". Pujo devait s'en féliciter par la suite, au moment de la création du Front de la liberté.

18. APP, 30 juin 1934, BA 1964.

19. APP, 24 décembre 1934, BA 1964.

20. APP, 25 janvier 1935, BA 1964.

21. APP, 11 novembre 1935, BA 1942. 
Il n'est pas étonnant, dans ces conditions, que les désaccords aient été fréquents entre l'Action française et ses alliés. L'Action française penchait facilement pour des initiatives de type " activiste ", destinées à maintenir la pression de la rue, de manière à ne laisser aucun répit aux gouvernements, même à ceux issus de l'Union nationale formée au lendemain du 6 février. En revanche, les ligues, membres à part entière du Front national, semblaient peu soucieuses de se laisser entraîner par la ligue royaliste. Ainsi, en janvier 1935, l' "Association des victimes du 6 février ", présidée par Louis Darquier de Pellepoix, proposa un défilé place de la Concorde pour célébrer le premier anniversaire de l'événement, ce qu'accepta volontiers le Front national qui s'en remit à l'Association pour l'organisation de la manifestation ${ }^{22}$. Mais les organisations contactées à l'extérieur du Front national ne manifestèrent aucun enthousiasme : ni les Croix-de-Feu, ni l'Union nationale des combattants n'acceptèrent de figurer dans le défilé. Pierre Taittinger, craignant l'isolement, opposa un refus à la proposition, suivi par la Solidarité française. L'Action française qui, dès le départ avait soutenu le projet, l'abandonna. D'autres frictions avaient déjà eu lieu : en juin 1934, la ligue royaliste avait reproché à ses partenaires du Front national de ne pas soutenir plusieurs de ses militants inculpés par le tribunal de Saint-Omer à la suite d'un affrontement dans le pays minier. Et par la suite, il y eut plus grave : en juin 1935, lors du renversement du gouvernement Flandin et de son remplacement par Pierre Laval, l'Action française tenta de provoquer des manifestations en sollicitant le Front national. Elle se heurta là aussi à un refus, et Trochu préféra suivre les conseils de modération du chef des Jeunesses patriotes.

Ces dissentiments posent deux catégories de problèmes, la signification de l'attitude des ligues membres du Front national, Jeunesses patriotes et Solidarité française; celle du comportement de Trochu ensuite. Les ligues associées dans le Front national se méfiaient manifestement de leur bruyant partenaire pour trois raisons. Une tenant à l'opportunité politique : elles redoutaient un noyautage de leurs troupes par l'AF, tout spécialement la Solidarité, entrée dans une crise profonde susceptible d'entraîner une partie de ses militants dans les rangs de la ligue royaliste. Une autre tenant au fond : le type d'action antiparlementaire envisagée en juin 1935 par l'AF entrait en contradiction formelle avec la position de Pierre Taittinger, qui, membre du groupe de la Fédération républicaine à la Chambre, adoptait une attitude de soutien et de participation au régime parlementaire. Il n'est pas exclu enfin que la crainte d'une législation hostile aux ligues activistes - adoptée en janvier 1936, puis appliquée d'abord contre l'Action française, ensuite par le Front populaire contre la Solidarité française, les Jeunesses patriotes et les Croix-de-Feu - ait contribué fortement à calmer les ardeurs des ligues associées dans le Front national.

Quant à Charles Trochu, il manifestait une évidente sympathie sinon pour l'Action française, au moins pour l'unité à la base, et dans le sens d'une

22. APP, 25 février 1935, BA 1964. 
plus grande radicalité. Ainsi, en février 1935, alors que les autorités religieuses refusaient l'absoute à un militant de l'Action française tué dans une rixe avec les communistes, le Front national appelait tous " les patriotes parisiens " à assister en masse aux obsèques ${ }^{23}$. En mai de la même année, le Front national accepta de s'associer à l'Action française pour le défilé en l'honneur de Jeanne d'Arc. Après la dissolution des organisations royalistes, Trochu accepta au printemps de 1936 de participer aux réunions d'un Comité national de vigilance de la jeunesse que tentaient de mettre sur pied les royalistes. Mais, au-delà de ces actions à caractère symbolique, Trochu ne put entraîner les ligues associées vers une attitude franchement activiste. Le Front national ne disparut pas avec le Front populaire. Lors de la création du Front de la liberté au printemps de 1936, les instances du Front national se réunirent pour décider que l'adhésion au nouveau rassemblement serait décidée par chaque composante ${ }^{24}$. En 1938, ce fut par l'intermédiaire de Trochu, encore secrétaire du Front national, que Maurice Pujo prit contact avec Louis Marin pour lui demander de participer comme représentant de la Fédération républicaine à une manifestation organisée en juin 1938, qu'il situait sur le plan du " patriotisme pratique ${ }^{25}$ ".

Au total, le bilan du premier "Front national " paraît bien limité. L'absence de programme, remplacé par quelques slogans portant sur l'antimaçonnisme, était bien révélateur des divergences existant entre les diverses organisations qui le composaient. Le Front national prit bien quelques initiatives débouchant sur des actions de type fusionnel, mais sans trouver de perspectives politiques bien claires. Son action, en définitive, ne devait avoir pour effet que de stimuler des attitudes militantes allant dans le sens d'une plus grande radicalité : si l'on en croit Jean Philippet, des comités locaux du Front national se seraient rapprochés de la Cagoule ${ }^{26}$ et des contacts auraient été pris avec l'UCAD en 1937. Coquille vide, puisque conçu comme un organe de liaison, le Front national ne put, malgré les efforts de son animateur, parvenir à accéder à une véritable existence politique, même si son action, à la base, put rassembler des militants d'origines diverses, inspirés par un même sentiment de radicalité.

\section{Une tentative de rapprochement des partis en 1937-1938 : le Front de la liberté}

Le " Front de la liberté " apparaît dans la phase de recomposition des forces de droite qui suivit la dissolution des ligues au cours de l'été 1936. Dans le courant de l'automne, on assista au développement rapide de partis nouveaux, le Parti social français (PSF) du colonel de La Rocque, héritier des Croix-de-Feu et le Parti populaire français (PPF) de Jacques Doriot,

23. APP, février 1935, BA 1964.

24. APP, 13 avril 1937, BA 1945.

25. Pujo à louis Marin, 29 mai 1938, AN, 317 AP 88.

26. PhILIPPET, Jean, op. cit., tome 4, p. 77. 
tandis que le Parti républicain national et social de Pierre Taittinger remplaçait les Jeunesses patriotes.

\section{Une mise sur pied difficile}

L'initiative est venue du PPF de Doriot, qui lança l'idée dès novembre $1936^{27}$ et formula une proposition concrète en mai 1937, à la veille de la chute du premier gouvernement de Front populaire. Le nom choisi par Doriot correspondait aux préoccupations du moment et fournissait un prétexte officiel à sa proposition. Il s'agissait, selon le fondateur du PPF, de défendre les libertés, bafouées, selon lui, par le gouvernement de Front populaire : la liberté de réunion, mise en cause, parfois en droit (interdiction par les autorités), quelquefois en fait (perturbations par les adversaires politiques); la liberté d'association, violée par les textes portant dissolution des ligues; enfin, la liberté du travail, constamment bafouée par les grévistes au cours des conflits du travail devenus incessants.

L'appel de Doriot s'adressait à un très large éventail de forces politiques, pratiquement tous les " nationaux ", c'est-à-dire tout ce qui était anticollectiviste. Le Chef du PPF envoya une lettre de proposition à neuf groupements : soit clairement situés dans la droite " dure ", comme le Parti social français du colonel de La Rocque, le Parti républicain national et social de Pierre Taittinger, le Parti agraire, soit relevant de la constellation parlementaire, à droite, comme la Fédération républicaine, au centre comme le Parti démocrate populaire et l'Alliance démocratique, ou à gauche, comme le Parti radical-socialiste et l'Union socialiste républicaine. Quelques jours plus tard, le 7 mai, au Vélodrome d'Hiver, le Chef du PPF précisa que « le respect des règles constitutionnelles de la III $^{\mathrm{e}}$ République " s'imposait nécessairement, car, ajouta-t-il, ces règles n'étaient pas si " désuètes et périmées " que d'aucuns voulaient le dire. Du coup, l'Action française se trouvait exclue du rassemblement dont Doriot prenait l'initiative. Écartés une fois de plus, au moins officiellement, parce que trop compromettants, les hommes d'Action française protestèrent par la plume de Maurice Pujo, qui énuméra dans L'Action française du 24 mai 1937 la longue liste de ces exclusions dans un article significativement intitulé « De Ba-Ta-Clan au Vel d'Hiv' ".

Mais l'appel de Doriot fut diversement entendu. Seuls se rendirent à une réunion de travail qu'il provoqua le 28 mai $^{28}$ des représentants de la Fédération républicaine (FR), du Parti républicain national et social (PRNS), du Parti social français (PSF) et du minuscule " Parti radical national ", dont les dirigeants, après quelques hésitations, déclinèrent l'offre quelques jours plus $\operatorname{tard}^{29}$. Auparavant, le conseil national du PSF réuni le 9 juin avait, sur l'insistance du colonel de La Rocque, refusé l'adhésion au Front de la liberté. Aussi le meeting inaugural du Front ne réunit-il, le 22 juin au Vel d'Hiv, que

27. Le Jour, 20 avril 1937.

28. APP, 28 mai 1937, BA 1945.

29. APP, 9 juin 1937, 12 juin 1937, BA 2002. 
des représentants du PPF, du PRNS, et de la Fédération républicaine qui avait chargé Xavier Vallat et Philippe Henriot de la représenter. Vallat prit soin de préciser que la FR n'était pas derrière Doriot mais à côté de lui ${ }^{30}$.

Ces difficultés de constitution étaient bien révélatrices des objectifs et des arrière-pensées propres à chaque composante.

\section{Des arrière-pensées politiques}

Derrière le prétexte respectable et consensuel - " défendre les libertés "-, se dissimulaient des objectifs stratégiques élaborés par des formations qui, anciennes ou récentes, vivaient difficilement la période de recomposition en cours. Doriot visait incontestablement la respectabilité politique, au premier chef auprès des " nationaux " de base. Beaucoup d'entre eux, et aussi les parlementaires de droite, ne voyaient en lui que l'ancien communiste condamné autrefois pour actes et propos antipatriotiques. Ainsi, quelques jours avant de lancer son appel du 7 mai, le Chef avait réussi à provoquer une réunion chez Charles des Isnards, député de Paris, un " héros " du 6 février, très proche des Jeunesses patriotes et de Taittinger ${ }^{31}$. Les parlementaires parisiens présents, Kerillis, Chiappe, Frédéric-Dupont, Taittinger lui-même, réservèrent leur réponse : sans doute le poids des Croix-de-Feu (dissous, mais regroupés dans le PSF) pesait-il sur cette attitude, mais la méfiance à l'égard de Doriot la déterminait aussi de façon décisive. La Rocque était sincère quand, devant le conseil national de son parti réuni le 9 juin, il déclara trouver le passé de Doriot " répugnant $^{32}$ ". Il n'est pas étonnant que Doriot ait, dans ces conditions, recherché l'appui d'un homme aussi " respectable " que Louis Marin. D'autres avantages pouvaient découler de cette insertion dans les forces établies de droite, comme l'appui de milieux financiers, dont l'aide au PPF se révélait irrégulière, bien que non négligeable ${ }^{33}$. Dans cette perspective, Doriot espérait faire figure de meilleur rempart contre le communisme ${ }^{34}$ et La Rocque ne se trompait pas quand il estimait que Doriot cherchait une place avantageuse dans la course aux subventions.

Mais il n'est pas douteux que Doriot poursuivait un autre objectif, la recherche de nouveaux adhérents pour son parti dont il voulait faire un mouvement de masse. Il comptait pour cela sur les sentiments unitaires des " nationaux " de base. De fait, il semble qu'un courant se dégagea au sein du PSF en faveur de l'adhésion, dans la perspective d'un rassemblement, d'un " front de tous les partis et de tous les groupements nationaux ${ }^{35}$ ". De même, des adhérents de la Fédération républicaine ne cachaient pas à Louis Marin

30. APP, 23 juin 1937, BA 2002.

31. APP, 26 avril 1937, BA 2002.

32. APP, 8 juin 1937, BA 2002.

33. APP, 4 mai 1937, BA 1945.

34. APP, 28 mai 1937, BA 1945.

35. APP, 8 juin 1937, BA 2002. 
leur souhait de voir se réaliser une sorte d'unité à la base ${ }^{36}$. L'évolution du Front allait bien confirmer cette visée de Doriot.

Mais que recherchait la Fédération de Louis Marin, vieille institution respectable, fondée en 1903 par un groupe de vieux et dignes républicains qui se réclamaient de Jules Méline et d'Alexandre Ribot? Pour la FR, il ne pouvait s'agir que d'une opération tendant à freiner l'inquiétante ascension du PSF. Il fallait faire en sorte que les troupes qui se portaient sur le PSF refluent vers le PPF et le Front de la liberté. Les dirigeants de la Fédération comptaient sur un courant unitaire de base qu'elle ne pouvait que difficilement capter, sa structure associative et élitaire lui interdisant - sauf le cas spécifique de la Fédération du Rhône - de se transformer franchement en parti de masse. Le drame pour la Fédération résidait dans l'apparition de conditions nouvelles dans les luttes politiques. Cette association s'était appuyée sur un réseau de notables alors en voie de dislocation, menacés par la montée des partis de masse, qui refusaient désormais de se laisser enfermer dans la distinction entre le "parti ", organe de l'élite élue ou éligible, et la " ligue ", bonne pour l'agitation de masse. Le Front de la liberté représentait un suprême effort pour freiner le PSF, l'alliance avec Doriot n'étant perçue que comme un expédient provisoire et comme un moindre mal. Quant au PRNS de Taittinger, il cherchait à retrouver la vitalité perdue des Jeunesses patriotes et comptait trouver là l'occasion de recruter de nouveaux adhérents ${ }^{37}$.

\section{Une structure rigoureusement confédérale et des modalités d'action assez limitées}

Dès le départ, le Front fut doté d'organes de liaison. Un comité de liaison au plan national semble avoir réuni hebdomadairement des représentants des composantes, soit le PPF, le PRNS, et la Fédération ${ }^{38}$. Celle-ci était représentée par quatre personnes : Xavier Vallat, Jacques Poitou-Duplessy, Camille Blaisot et Jean Guiter. Pour qui connaît un peu la FR de l'intérieur, ces choix étaient significatifs. À côté d'un " ligueur " (Vallat), figuraient deux hommes d'appareil, Poitou et surtout Guiter, le secrétaire général du parti, qui jouissait de la confiance totale de Marin, et un leader parlementaire, ancien ministre, Camille Blaisot. Parmi les représentants des autres organisations figuraient Claude Popelin, chef de la propagande du PPF et René Richard délégué général du PRNS. Mais cela ne déboucha nullement sur la constitution d'organisations politiques permanentes réunissant dans les départements les trois groupes adhérents. La Fédération républicaine s'y opposa formellement, n'admettant que des organisations - bien hypothétiques! - qui regrouperaient la totalité des partis " nationaux ": outre les

36. Lettres de plusieurs membres sociétaires, un colonel en retraite, un directeur d'aciérie, AN, 317 AP 88.

37. APP, 2 juin 1937, BA 2002.

38. APP, 2 novembre 1937, BA 1951, confirmé par Guiter à Marin, 17 décembre 1937, AN, 317 AP 72. 
trois composantes, l'Alliance démocratique, le Parti démocrate populaire, les radicaux indépendants, le PSF éventuellement. Le secrétaire général de la FR veilla à ce que ne se constitue pas un « "super-parti”, susceptible d'aliéner en quoi que ce soit la liberté d'organisation, de propagande ou d'action de chacun des groupements participants ${ }^{39}$ ".

Dans ces conditions, les modalités d'action communes restèrent bien limitées. Marin avait bien tenté au départ de formuler des propositions dans le domaine programmatique, mais cette suggestion fut écartée par Doriot, qui ne souhaitait se lier en ce domaine en aucune façon ${ }^{40}$. Restaient deux domaines d'action commune, la propagande et l'alliance électorale. Des réunions communes furent organisées par le Front de la liberté, notamment en Loire-inférieure (juillet), à Annonay (août), à Neuilly (novembre). Plusieurs traits ressortent de ces réunions. Elles se tinrent devant des auditoires bien étrangers au groupement qu'était le PPF : les paysans de la Basse Loire et ceux de l'Ardèche répondaient à l'appel de leurs notables (à Nantes, assistèrent au meeting de La Ferronays, de Juigné, Le Cour Grandmaison, ainsi que le comte de Suzannet; Annonay était le fief de Vallat). Les dirigeants nationaux de la FR furent dans l'ensemble assez discrets, et Marin ne parut guère sur les estrades du Front de la liberté. Il semblait en effet aux dirigeants de la FR que la présence de leur président, qui était également chef de groupe parlementaire, aux côtés des chefs du PRNS et du PPF pouvait entraîner des inconvénients politiques : si Doriot pensait acquérir une certaine respectabilité, Marin pouvait perdre la sienne. Il s'agissait aussi et surtout de ménager les alliés centristes de la Fédération ${ }^{41}$. Enfin, dans la période de dislocation du Front, à la fin de 1937, le déroulement des réunions lui-même montrait bien le caractère disparate de l'alliance. Ainsi, à Neuilly, en novembre, dans une réunion dont Kerillis avait accepté la présidence, à la fin du meeting, les divers groupes représentés, PPF, PRNS et ligueurs de l'ex-Action française qui étaient venus en nombre, entonnèrent l'hymne de leur parti respectif après avoir chanté en chœur " la Marseillaise ${ }^{42}$ ". Les services d'ordre, comme pour le Front national étaient fournis par chaque groupe, l'Action française jouant encore un rôle important attesté à plusieurs reprises.

Plus ambitieux, et sans doute figurant parmi les priorités des dirigeants de la Fédération, un protocole d'accord en vue des élections cantonales de septembre 1937 fut conclu entre les composantes ${ }^{43}$. Il fut, aux termes de ce texte, décidé que la procédure d'accord entre les groupes nationaux se feraient en deux temps. Il y aurait d'abord, au préalable un accord entre les trois composantes du Front de la liberté. Puis, le délégué de la Fédération serait chargé d'entrer en contact avec les autres groupes nationaux

39. Guiter à Marin, 17 décembre 1937, AN, 317 AP 72.

40. APP, 8 mai 1937, BA 2002.

41. AN, Guiter à Marin, 20 décembre 1937, AN, 317 AP 72.

42. APP, 9 novembre 1937, BA 2002.

43. Circulaire Guiter, 3 août 1937, AN, 317 AP 88. 
(Alliance démocratique, Parti démocrate populaire, radicaux indépendants, mais non le PSF, avec qui des discussions pourraient être engagées dans une ultime phase) en vue d'une candidature unique; à défaut, si cela semblait plus " opportun ", un délégué du PPF ou du PRNS serait chargé de la discussion. Ce protocole semblait une des priorités du vieux parti parlementaire, et les élections de septembre 1937 montrèrent une certaine discipline chez les «nationaux ». Mais la FR avait disposé de l'avantage de l'ancienneté et d'une implantation notabiliaire plus importante que les autres composantes.

\section{Des relations fondées sur l'intérêt respectif et la méfiance réciproque}

Dès le lendemain des cantonales, dans les dernières semaines de 1937, les relations se tendirent entre les partenaires du Front de la liberté. Deux types d'agissements furent alors dénoncés par Guiter. D'une part, l'organisation de conférences dites du " Front de la liberté " par les dirigeants PPF de certains départements sans l'avis du comité local de la Fédération. Dans cette pratique, Guiter voyait l'indice d'une tentative de création de comités "Front de la liberté " autonomes, dans lesquels seraient noyés les représentants de la Fédération. Il lui parut donc nécessaire de faire une ferme mise au point sur la totale indépendance des composantes, dont les membres ne devaient dépendre que de leur comité directeur central respectif. Il semble donc bien - sous réserve de vérification - que le PPF ait cherché à transformer le Front de la liberté en réseau autonome, doté d'un pouvoir de décision propre par rapport à ses composantes, dont Doriot aurait pu ensuite prendre le contrôle. Cette pratique, du reste, correspondait bien à l'objectif affiché au départ. Pour autant, il faut souligner que la FR a sûrement accéléré la rupture, choisissant le premier prétexte venu. L'alliance avec le PPF pouvait en effet, une fois les cantonales passées, gêner ses rapports avec les formations " centristes" (Alliance démocratique et PDP), alors que leur appui paraissait indispensable dans les campagnes législatives à venir.

Aussi, dès décembre 1937, Guiter fit ralentir la participation de la FR aux manifestations à venir du Front de la liberté, tout en laissant aux fédérations départementales le soin de poursuivre localement leur collaboration, si elles estimaient cela nécessaire. Mais en aucun cas, elle ne pourrait être poursuivie si elle était de nature à " gêner " les militants locaux ou les parlementaires " $\operatorname{amis}^{44}$ ". Quant à Marin, il opta au début de 1938 pour une abstention totale. Le secrétaire adjoint de la Fédération déclina en son nom en février 1938 une invitation à participer en Savoie à une réunion commune avec le PPF et le PRNS, Marin ayant fait savoir qu'il ne souhaitait nullement " figurer sur une estrade " aux côtés des présidents de ces deux formations ${ }^{45}$.

44. Guiter à Marin, 20 décembre 1937, AN, 317 AP 72.

45. Guiter à Marin, 20 décembre 1937 et 9 février 1938, AN, 317 AP 72. 
Le Front de la liberté peut donc être considéré comme une combinaison mise au point exclusivement par des organisations partisanes. Le stade des ligues, confusément fusionnel, était dépassé. Il s'agissait là d'une opération montée par deux acteurs principaux, le PPF et la Fédération républicaine, pour faire face à une situation difficile dans un contexte d'affrontement et de recomposition politique, la mise au point d'une organisation confédérale ayant pour objectif essentiel de freiner l'ascension d'un rival jugé dangereux. Son existence ne pouvait donc être que temporaire, surtout pour la formation la plus préoccupée par les considérations électorales immédiates, la Fédération républicaine.

Les tentatives de regroupement des forces de droites ont donc été circonstancielles, entreprises pour une brève durée. Elles mettent en valeur un fait bien connu : les rivalités personnelles, les divergences idéologiques, les conflits d'intérêt, qui rendaient impossible un rapprochement durable. À quoi s'ajoutait une distorsion importante entre une base souvent " unitaire ", animée par un sentiment confusément fusionnel, surtout chez les militants de la rue, et des états-majors beaucoup plus réticents. Deux points toutefois méritent d'être soulignés.

1- Ces tentatives ont toujours été le fait de groupes minoritaires, ou se percevant comme tels. L'Action nationale républicaine se voulait le pendant des formations de gauche, radicale et socialiste, réputées plus structurées, et dont on redoutait la victoire aux élections de 1924, et son souci principal était d'éviter une marginalisation de la droite pure par des formations centristes comme l'Alliance démocratique. Les ligues des années 1930 qui s'unirent dans le Front national étaient alors en perte de vitesse, de même que la Fédération républicaine qui choisit de collaborer avec le PPF pour contrer l'irrésistible ascension du colonel de La Rocque. Ces expériences témoignent en fait de l'extraordinaire plasticité de la vie politique française, toute apparition d'une puissance hégémonique aboutissant à la constitution d'une coalition de forces hostiles.

2- Ces tentatives correspondirent à des âges différents de la vie politique. Les créateurs de l'ANR espéraient dépasser le stade des associations à base exclusivement parlementaire, de même que ceux de la Ligue républicaine nationale, en introduisant des non parlementaires. Ils ne parvinrent qu'à créer quelques contacts entre des notables dont les préoccupations se limitaient à celles du monde parlementaire. Bien différent était le monde des ligues des années 1930. Ici, la préoccupation principale était le recrutement de masse, en vue de créer une visibilité dans la rue. Plus profondément, il s'agissait de faire surgir dans les masses un " peuple de droite ", comme surgissait alors un peuple de gauche qui s'unissait dans le cadre du Front commun. Enfin, le Front de la liberté, misérable marché de dupes, n'en laissait pas moins apparaître le rôle des partis, par ce curieux dialogue qui s'établit entre le PPF, parti « moderne " - ou qui se présentait comme tel - et la vieille association de notables qui tentait, non sans habileté, de perpétuer son organisation à base notabiliaire et parlementaire. 


\section{RESUME}

Dans les année 1930, on assiste à plusieurs tentatives de mise sur pied par des groupes de droite d'organisations de type " confédéral ". Elles empruntent peu aux ligues des années 1920, comme l'Action Nationale républicaine, coalition essentiellement formée de groupes dirigés par les parlementaires. Mais le Front national (créé en 1934) ne dépassa jamais le stade d'organe de liaison entre les ligues déclinantes. Quant au Front de la liberté (créé en 1937), composé essentiellement de la Fédération républicaine et du Parti populaire français, il ne fut qu'une tentative pour freiner l'ascension du Parti social français du colonel de La Roque.

\section{ABSTRACT}

The attempts at uniting the rightist parties in the thirties

In the nineteen thirties we know several attempts at creating organizations on the "confederal" type by rightist groups. They borrow little from the leagues of the twenties, such as the Action Nationale républicaine, a coalition essentially made of groups led by the parlementarians. But the Front national (created in 1934) never went beyond the stage of organ of connection between the declining leagues. As for the Front de la Liberte (created in 1937) essentially composed of the Fédération républicaine and the Parti populaire français, it was only an attempt in order to slow down the ascension of the Parti social français of the Colonel de La Roque. 
